\title{
Nonlocal in time problem for evolution pseudodifferential equations with analytic symbols
}

\author{
V. V. Gorodetsky, A. A. Shyrokovskykh \\ Yuriy Fedkovych Chernivtsi National University, Chernivtsi, Ukraine \\ Corresponding author. E-mail: a.shyrokovskykh@gmail.com
}

Paper received 24.02.20; Accepted for publication 16.03.20.

\section{https://doi.org/10.31174/SEND-NT2020-233VIII28-06}

\begin{abstract}
The definitions and properties of fundamental solution for nonlocal multi-point in time problem for evolution equations with pseudodifferential operators constructed at variable symbols is given. The solvability of multi-point problem in the $W$ type spaces is installed and the integral image interpretation of solution is given.

Keywords: evolution equation, pseudodifferential operator, nonlocal problem, Fourier transform.
\end{abstract}

Introduction. A rather broad class of differential equations with partial derivatives are linear parabolic and $B$ parabolic equations, whose theory originates from the investigation heat equation. The classical theory of the Cauchy problem and boundary-value problems for such equations and systems of equations is constructed in the works of I.G. Petrovsky, S.D. Eidelman, S.D. Ivashyshen, M.I. Matiychuk, M.V. Zhytarash, A. Friedman, S. Teklind, V.O. Solonnikov, V.V. Krehivsky and others. The Cauchy problem with initial data from the spaces of generalized functions of the type of distributions and ultra-distributions was studied by G.E. Shilov, B.L. Gurevich, M.L. Gorbachuk, V.I. Gorbachuk, O.I. Kashpirovsky, Ya.I. Zhytomyrsky, S.D. Ivashyshenym, V.V. Gorodetsky, V.A. Litovchenko, etc.

A formal extension of the class of parabolic type equations is the evolution equations with pseudodifferential operators (PDOs), which can be represented as $A=$ $I_{\sigma \rightarrow x}^{-1}\left[a(t, x ; \sigma) I_{x \rightarrow \sigma}\right], \quad\{x, \sigma\} \subset \mathbb{R}^{n}, t>0$, where $a$ is a function (symbol) that satisfies certain conditions, $I, I^{-1}$ is a direct and inverse Fourier or Bessel transform. PDOs include differential operators, fractional differentiation and integration operators, convolution operators, Bessel operator $B_{v}=d^{2} / d x^{2}+(2 v+1) x^{-1} d / d x, \quad v>-1 / 2$, which in its structure contains the expression $1 / x$ and formally represented as $B_{v}=F_{B_{v}}^{-1}\left[-\sigma^{2} F_{B_{v}}\right]$, where $F_{B_{v}}$ is the integral transformation of Bessel and others.

Today, in the theory of the Cauchy problem for evolutionary pseudodifferential equations in questions of the correct solvability of the Cauchy problem, the image of the solution in the case where the initial conditions are elements of different functional spaces (in particular, the spaces of generalized functions), significant results are obtained, native and foreign mathematicians (M. Nagase, R. Shinkai, C. Tsutsumi, Yu.A. Dubinsky, S.D. Eidelman, M.V. Fedoryuk, Y.M. Dryin, V.V. Gorodetsky, etc.).

One of the generalizations of the Cauchy problem for partial differential equations is the nonlocal multi-point in time problem, where the initial condition $\left.u(t, \cdot)\right|_{t=0}=f$ is replaced by the condition $\left.\sum_{k=0}^{m} \alpha_{k} u(t, \cdot)\right|_{t=t_{k}}=f$, where $t_{0}=0,\left\{t_{1}, \ldots, t_{m}\right\} \subset(0, T],\left\{\alpha_{0}, \alpha_{1}, \ldots, \alpha_{m}\right\} \subset \mathbb{R}, m \in$ $\mathbb{N}$ - are fixed numbers (if $\alpha_{0}=1, \alpha_{1}=\alpha_{2}=\ldots=\alpha_{m}=$ 0 , then obviously we have a Cauchy problem.) Nonlocal in time problems refer to nonlocal boundary-value problems for equations with partial derivatives. Nonlocal problems arise when modeling different processes and practices of boundary-value problems for equations with partial derivatives with nonlocal conditions (see. eg., [1, 2]).

Many mathematicians have been involved in the study of nonlocal boundary value problems using different methods and approaches (see, for example, [3-11]). Important results were obtained concerning the formulation, correct solvability and solution construction, and the conditions of regularity of boundary conditions were formulated for important cases of differential operator equations.

This paper investigates a nonlocal multi-point in time problem for evolution equations with pseudodifferential operators constructed at variable symbols by Fourier transform. The analytic function of a pseudodifferential operator symbol character makes it possible to understand such an operator as an infinite-order differentiating operator with variable coefficients acting in a certain space of analytic functions. This gives a definition of the fundamental solution of the specified problem and investigates the properties of such a solution, establishes the solvability of the multi-point problem. An integral image of the solution is found.

Previous data. Consider the function $\omega:[0,+\infty) \rightarrow$ $[0,+\infty)$, which is continuous and increasing, with $\omega(0)=$ $0, \lim _{x \rightarrow+\infty} \omega(x)=+\infty$. Set $\Omega(x)=\int_{0}^{x} \omega(\xi) d \xi$ for $x \geq 0$. The function $\Omega$ has the following properties:

1) $\Omega$ is a differential function increasing by $[0,+\infty)$, and $\Omega(0)=0, \lim _{x \rightarrow+\infty} \Omega(x)=+\infty$;

2) $\Omega$ is a convex downward function $[12$, p. 8], that is $\forall\left\{x_{1}, x_{2}\right\} \subset[0,+\infty): \Omega\left(x_{1}\right)+\Omega\left(x_{2}\right) \leq \Omega\left(x_{1}+x_{2}\right)$.

We define the function $\Omega$ on $(-\infty, 0]$ in an even way. Next, consider the function $\mu:[0,+\infty) \rightarrow[0,+\infty)$, which has the same properties, like the $\omega$ function. Set $M(x)=$ $\int_{0}^{x} \mu(\xi) d \xi, M(-x)=M(x)$ for $x \geq 0$. Using the functions $M$ and $\Omega$, B.L. Gurevich [13] introduced a series of spaces, which he called spaces $W$. Here are definitions of some of these spaces.

The space $W_{M}^{\Omega}$ is constructed by the functions $\Omega$ and $M$ and is defined as the set of integer functions $\varphi: \mathbb{C} \rightarrow \mathbb{C}$ that satisfy the inequality $|\varphi(z)| \leq \tilde{c} \exp \{-M(\tilde{a} x)+$ $\Omega(\tilde{b} y)\}, z=x+i y$, with some positive constants $\tilde{c}, \tilde{a}, \tilde{b}$, dependent only on $\varphi$. $W_{M}^{\Omega}$ can be represented as a union of count-normalized spaces $W_{M, a}^{\Omega, b}$, where $W_{M, a}^{\Omega, b}$ consists of those functions $\varphi \in W_{M}^{\Omega}$ for which the inequalities

$$
\begin{gathered}
|\varphi(x+i y)| \leq \tilde{c} \exp \{-M(\bar{a} x)+\Omega(\bar{b} y)\}, \\
z=x+i y \in \mathbb{C},
\end{gathered}
$$

where $\bar{a}$ is an arbitrary positive constant less than $\tilde{a}$ and 
$\bar{b}$ is arbitrarily constant greater than $\tilde{b}$. If for $\varphi \in W_{M, a}^{\Omega, b}$ we set

$$
\begin{gathered}
\|\varphi\|_{\delta \rho}=\sup _{z \in C}[|\varphi(z)| \times \\
\times \exp \{-\Omega((\tilde{b}+\rho) y)+M(\tilde{a}(1-\delta) x)\}], \\
\{\delta, \rho\} \subset\{1 / n, n \geq 2\},
\end{gathered}
$$

then with these norms the space $W_{M, a}^{\Omega, b}$ becomes a complete perfect countable-normalized space [12, p. 16] Combining the spaces $W_{M, a}^{\Omega, b}$ over all $a=1, \frac{1}{2}, \ldots$ and $b=$ $1,2, \ldots$ matches the space $W_{M}^{\Omega}$.

The spaces $W$ are taken by the Fourier transform to spaces of the type $W$. To state a related assertion, we introduce the notion of Young dual functions.Let $M(x)$ and $\Omega(y)$ be functions defined via the functions $\mu(\xi)$ and $\omega(\eta)$ respectively. If the functions $\mu$ and $\omega$ are mutually inverted, that is, $\mu(\omega(\eta))=\eta$ and $\omega(\mu(\xi))=\xi$, then the functions $M(x)$ and $\Omega(y)$ are said to be Young dual. Examples of mutually dual functions are the functions

$$
M(x)=x^{p} / p, \Omega(y)=y^{q} / q, 1 / p+1 / q=1 .
$$

We denote by $W_{M}^{\Omega}(\mathbb{R})$ the set of functions given by $\mathbb{R}$, which are narrowing functions from $W_{M}^{\Omega}$ by $\mathbb{R}$. The correct formula is $\left[12\right.$, p. 32]: $F\left[W_{M}^{\Omega}(\mathbb{R})\right]==W_{M_{1}}^{\Omega_{1}}(\mathbb{R})$, where $F$ is the Fourier transform, $\Omega_{1}$ and $M_{1}$-functions Young dual to the functions $M$ and $\Omega$, respectively.

For arbitrarily fixed $\alpha, \beta>0$ we set

$$
\begin{gathered}
S_{\alpha}^{\beta}(\mathbb{R}) \equiv S_{\alpha}^{\beta}:=\left\{\varphi \in C^{\infty}(\mathbb{R}) \mid \exists c, A, B>0\right. \\
\forall\{k, n\} \subset \mathbb{Z}_{+} \forall x \in \mathbb{R}: \\
\left.\left|x^{k} \varphi^{(n)}(x)\right| \leq c A^{k} B^{n} k^{k \alpha} n^{n \beta}\right\} .
\end{gathered}
$$

The spaces introduced can be characterized as [14, p. 210]. The spaces $S_{\alpha}^{\beta}$ are non-trivial at $\alpha+\beta \geq 1$ and form dense sets in $L_{2}(\mathbb{R}) . S_{\alpha}^{\beta}$ consists of those and only those functions $\varphi \in C^{\infty}(\mathbb{R})$, the inequality $\left|\varphi^{(n)}(x)\right| \leq c B^{n} n^{n \beta} \exp \left(-a|x|^{1 / \alpha}\right), n \in \mathbb{Z}_{+}, x \in \mathbb{R}$,

hold with some positive constants $c, a, B$ dependent on the function $\varphi$.

If $0<\beta<1$ and $\alpha \geq 1-\beta$, then $S_{\alpha}^{\beta}$ consists of those and only functions $\varphi \in C^{\infty}(\mathbb{R})$ which analytically continued in the complex plane and satisfying inequality

$$
\begin{gathered}
|\varphi(x+i y)| \leq c \exp \left(-a|x|^{1 / \alpha}+b|y|^{1 /(1-\beta)}\right), \\
c, a, b>0 .
\end{gathered}
$$

Note that $S_{\alpha}^{\beta} \equiv W_{M}^{\Omega}$, where $M(x)=x^{1 / \alpha}, \Omega(y)=$ $y^{1 /(1-\beta)}, 0<\alpha<1,0<\beta<1, \alpha+\beta \geq 1$.

The spaces of $S_{\alpha}^{\beta}$ by Fourier transforms are reflected in spaces of the same type, namely, the formula is correct [14, p. 245]: $F\left[S_{\alpha}^{\beta}\right]=S_{\beta}^{\alpha}$.

Problem statement. Consider the function $a(t, x ; \sigma)$ given by $[0, T] \times \mathbb{R} \times \mathbb{R}$ which satisfies the conditions:

1) $a(t, x ; \sigma)$ is a continuously differentiating function of the argument $t \in[0, T]$ (for fixed $x, \sigma) ; a(t, x ; \sigma)$ is a continuously differentiable function of $x$ (for fixed $t, \sigma$ ) bounded by $\mathbb{R}$;

2 ) for fixed $t, x$, the function $a(t, x ; \sigma)$, as a function of variable $\sigma$, permits analytic extension into the whole complex plane, while

$$
\begin{gathered}
\forall \varepsilon>0 \quad \exists c_{\varepsilon}>0 \quad \forall \sigma+i \tau \in \mathbb{C}: \\
|a(t, x ; \sigma+i \tau)| \leq c_{\varepsilon} \exp \{M(\varepsilon \sigma)+\Omega(\varepsilon \tau)\}, \\
\forall(t, x) \in \Pi_{T} \equiv[0, T] \times \mathbb{R}
\end{gathered}
$$

(ie, $a(t, x ; \cdot)$ multiplier in $W_{M}^{\Omega}$ space);

$$
\exists c, a, b>0 \text { : }
$$

$$
\begin{gathered}
|\exp \{a(t, x ; \sigma+i \tau)\}| \leq \exp \{-M(a \sigma)+\Omega(b \tau)\}, \\
\forall(t, x) \in \Pi_{T}
\end{gathered}
$$$$
\text { (ie, } \left.\exp \{a(t, x ; \cdot)\} \in W_{M}^{\Omega}\right) \text {. }
$$

We also consider that $M$ satisfies the condition: $\exists c_{0}>$ $0 \forall x \in \mathbb{R}: M(x) \geq c_{0}|x|^{\alpha}$, with fixed parameter $\alpha>2$.

Consider the pseudodifferential operator $A$, constructed by a symbol $a(t, x ; \sigma)$ :

$$
\begin{gathered}
(A \psi)(x):=F_{\sigma \rightarrow x}^{-1}\left[a(t, x ; \sigma) F_{x \rightarrow \sigma}[\psi(x)](\sigma)\right](x), \\
\forall \psi \in W_{M_{1}}^{\Omega_{1}}(\mathbb{R}),
\end{gathered}
$$

where $M_{1}, \Omega_{1}$ - functions Young dual to the functions $M$ and $\Omega$, respectively.From the properties of the functions $a(t, x ; \sigma)$, it follows that $\quad A \psi \in K(\mathbb{R})$ for every $t \in[0, T]$, where $K(\mathbb{R})$ is a normalized space consisting of continuous functions $\varphi$ bound for $\mathbb{R}$ with $\|\varphi\|=\sup _{x \in \mathbb{R}}|\varphi(x)|$. Note also that $A$ can be understood as the operator of differentiation infinite order (see. [15]), ie $A=\sum_{k=0}^{\infty} c_{k}(t, x)\left(-i D_{x}\right)^{k}$, provided that $a(t, x ; \sigma)=$ $\sum_{k=0}^{\infty} c_{k}(t, x) \sigma^{k}$ is the Taylor series of the function symbol $a$ by the variable $\sigma$ (at the fixed $t, x$ ).

In the band $\Pi^{\prime}{ }_{T}=\{(t, x): 0 \leq \tau<t \leq T, \quad x \in \mathbb{R}\}$ we consider the problem of finding the solution of the evolution equation

$$
\partial u(t, x) / \partial t=A u(t, x), \quad(t, x) \in \Pi_{T}^{\prime},(1)
$$

which satisfies the conditions:

$$
\begin{gathered}
u(t, x)=u_{1}(t, x)+u_{2}(t, x), \\
\mu \lim _{t \rightarrow \tau+0} u_{1}(t, x)-\sum_{\substack{m \\
\lim _{t \rightarrow \tau+0} u_{2}(t, x)=0,(3)}} \mu_{k} \lim _{t \rightarrow t_{k}} u_{1}(t, x)=\varphi(x),
\end{gathered}
$$

at each point $x \in \mathbb{R}$ for the fixed function $\varphi \in$ $W_{M_{1}}^{\Omega_{1}}(\mathbb{R}), \quad m \in \mathbb{N}, \quad\left\{\mu, \mu_{1}, \ldots, \mu_{m}\right\} \subset(0,+\infty)$, $\left\{t_{1}, \ldots, t_{m}\right\} \subset(\tau, T]$ are fixed numbers, and $\mu>$ $m \sum_{k=1}^{m} \mu_{k}, \quad 0 \leq \tau<t_{1}<\ldots<t_{m}=T$. Problem (1)-(3) is called nonlocal $m$-point (multi-point) in time problem for equation (1).

Main results. At a fundamental solution of the problem (1)-(3) we understand the function

$$
\begin{array}{r}
Z(t, x ; \tau, \xi)=V(t, x ; \tau, \xi)+\Gamma(t, x ; \tau, \xi), \\
(t, x) \in \Pi^{\prime}, \quad 0 \leq \tau<t \leq T, \quad \xi \in \mathbb{R},
\end{array}
$$

which has the properties of:

1) $L Z(t, x ; \tau, \xi)=0, L \equiv L(t, x ; A, \partial / \partial t):=\quad=\partial /$ $\partial t-A$, ie $Z$, as a function of $(t, x)$ (at the fixed $\tau, \xi$ ) is the solution of equation (1);

$$
\begin{aligned}
&2) \lim _{t \rightarrow \tau+0} \int_{\mathbb{R}} V(t, x ; \tau, \xi) \varphi(\xi) d \xi- \\
&-\sum_{k=1}^{m} \mu_{k} \lim _{t \rightarrow t_{k}} \int_{\mathbb{R}} V(t, x ; \tau, \xi) \varphi(\xi) d \xi=\varphi(x), \\
& \lim _{t \rightarrow \tau+0} \int_{\mathbb{R}} \Gamma(t, x ; \tau, \xi) \varphi(\xi) d \xi=0
\end{aligned}
$$

at each point $x \in \mathbb{R}$ for an arbitrary function $\varphi \in$ $W_{M_{1}}^{\Omega_{1}}(\mathbb{R})$.

To construct the function $Z$, we use the Levi method (by parametrix). To this end, we fix $a(t, x ; \sigma)$ at $(t, x)=$ $(\chi, \xi), \quad \chi \in[\tau, T], \xi \in \mathbb{R}$ and consider the $m$-point problem for evolutionary equations with constant symbol $a(\chi, \xi ; \sigma)$ :

$$
L(\chi, \xi ; A, \partial / \partial t) v(t, x)=0, \quad(t, x) \in \Pi_{T}^{\prime},(4)
$$




$$
\begin{gathered}
\mu \lim _{t \rightarrow \tau+0} v(t, x)-\sum_{k=1}^{m} \mu_{k} \lim _{t \rightarrow t_{k}} v(t, x)=\varphi(x),(5) \\
\varphi \in W_{M_{1}}^{\Omega_{1}}(\mathbb{R}) .
\end{gathered}
$$

Solution $v \in C^{1}\left((\tau, T], W_{M_{1}}^{\Omega_{1}}(\mathbb{R})\right.$ of problem (4), (5) will be sought by means of the Fourier transform. We find directly, that

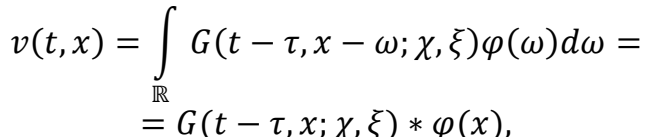

$$
\begin{aligned}
& G(t-\tau, x ; \chi, \xi)=F_{\sigma \rightarrow x}^{-1}[Q(t-\tau, \chi ; \xi, \sigma)], \\
& Q(t-\tau, \chi ; \xi, \sigma)=\exp \left\{\left(t_{k}-\tau\right) a(\chi, \xi ; \sigma)\right\} \times \\
& \times\left(\mu-\sum_{k=1}^{m} \mu_{k} \exp \left\{\left(t_{k}-\tau\right) a(\chi, \xi ; \sigma)\right\}\right)^{-1} .
\end{aligned}
$$

The properties of function $G$ depend on the properties of function $Q$, since $G=F^{-1}[Q]$. Using the results obtained in [16, p. 192], we come to the statement that there exists $c, a, b>0$, which are independent of $t, \tau, \chi, \xi$ such that for $Q$ and its derivatives (by the variable $\sigma$ ) estimates are valid

$$
\begin{gathered}
\left|D_{\sigma}^{n} Q(t-\tau, \chi ; \xi, \sigma)\right| \leq c\left(\frac{b e}{\rho_{n}}\right)^{n} n ! e^{-(t-\tau) M(a \sigma)}, \\
n \in \mathbb{Z}_{+}, \quad \sigma \in \mathbb{R},
\end{gathered}
$$

where $\rho_{n}$ is a solution of the equation $\sigma \omega(\sigma)=n, n \in$ $\mathbb{Z}_{+}, \omega=\Omega^{\prime}$.

Due to the Stirling formula

$n !=\sqrt{2 \pi n} n^{n} e^{-n} e^{\theta /(12 n)}, 0<\theta<1$. Then, using (6) and the estimate $M(\sigma) \geq c_{0}|\sigma|^{\alpha}, \sigma \in \mathbb{R}$, find that

$$
\begin{gathered}
\left|D_{\sigma}^{q} Q(t-\tau, \chi ; \xi, \sigma)\right| \leq c e \sqrt{2 \pi} \sqrt{q}\left(\frac{q}{e}\right)^{q}\left(\frac{b e}{\rho_{q}}\right)^{q} \times \\
\quad \times \exp \left\{-(t-\tau) \tilde{c}_{0}|\sigma|^{\alpha}\right\} \leq \\
\leq c_{2} b^{q} q^{q} \exp \left\{-\tilde{c}_{0}(t-\tau)|\sigma|^{\alpha}\right\}, \quad q \in \mathbb{Z}_{+},
\end{gathered}
$$

where $c_{2}=c c_{1} e \sqrt{2 \pi}$ (the properties of the sequence $\left\{\rho_{q}, q \in \mathbb{Z}_{+}\right\}$are taken into account here; see [16, p. 168]). We are directly convinced that the true inequality

$$
\begin{gathered}
\left|\sigma^{k} \exp \left\{-c_{0}^{\prime}(t-\tau)|\sigma|^{\alpha}\right\}\right| \leq(t-\tau)^{-k / \alpha} B^{k} k^{k / \alpha}, \\
k \in \mathbb{Z}_{+},
\end{gathered}
$$

where $c_{0}^{\prime}=\tilde{c}_{0} / 2, B=\left(\alpha \tilde{c}_{0}{ }^{\prime} e\right)^{-1 / \alpha}$. From the last inequality follow the estimates

$$
\begin{aligned}
& \left|\sigma^{k} D_{\sigma}^{q} Q(t-\tau, \chi ; \xi, \sigma)\right| \leq \\
\leq & c_{2} B^{k}(t-\tau)^{-k / \alpha} k^{k / \alpha} b^{q} q^{q} \times \\
& \times \exp \left\{-c_{0}^{\prime}(t-\tau)|\sigma|^{\alpha}\right\} .(7)
\end{aligned}
$$

Based on the estimates (7), we conclude that at $t>\tau$ the function $Q$, as a function of the argument $\sigma$, is an element of the space $S_{1 / \alpha}^{1}$.

Next, we use the ratios

$$
\begin{gathered}
x^{q} D_{x}^{k} F[\varphi](x)=i^{k+q} F\left[\left(\sigma^{k} \varphi(\sigma)\right)^{(q)}\right]= \\
=i^{k+q} \int_{\mathbb{R}}\left(\sigma^{k} \varphi(\sigma)\right)^{(q)} e^{i x \sigma} d \sigma, \\
\{k, q\} \subset \mathbb{Z}_{+}, \varphi \in S_{1 / \alpha}^{1} .
\end{gathered}
$$

Consequently,

$$
\begin{aligned}
& x^{q} D_{\chi}^{k} G(t-\tau, x ; \chi, \xi)=(2 \pi)^{-1}(-1)^{q} i^{k+q} \times \\
& \quad \times \int_{\mathbb{R}}\left(\sigma^{k} Q(t-\tau, \chi ; \xi,-\sigma)\right)^{(q)} e^{-i x \sigma} d \sigma .
\end{aligned}
$$

From the results given in $[14$, p. 243] implies that the double sequence $m_{k q}=k^{k / \alpha} q^{q},\{k, q\} \subset \mathbb{Z}_{+}$, satisfies the inequality

$$
k q \frac{m_{k-1, q-1}}{m_{k q}} \leq \gamma(k+q), \quad \gamma>0 .
$$

Then, applying the Leibniz formula for the product differentiation of two functions, the estimate (7) and the last inequality we find that

$$
\begin{aligned}
& \left|\left(\sigma^{k} Q(t-\tau, \chi ; \xi,-\sigma)\right)^{(q)}\right|= \\
& =\left|\sum_{p=0}^{q} C_{q}^{p}\left(\sigma^{k}\right)^{(p)} Q^{(q-p)}(t-\tau, \chi ; \xi,-\sigma)\right| \leq \\
& \leq\left|\sigma^{k} Q^{(q)}(t-\tau, \chi ; \xi,-\sigma)\right|+ \\
& +k q\left|\sigma^{k-1} Q^{(q-1)}(t-\tau, \chi ; \xi,-\sigma)\right|+\frac{k(k-1)}{2} \times \\
& \times q(q-1)\left|\sigma^{k-2} Q^{(q-2)}(t-\tau, \chi ; \xi,-\sigma)\right|+\ldots \leq \\
& \leq c_{2}\left[B^{k} b^{q} m_{k q}(t-\tau)^{-k / \alpha}+\right. \\
& +k q B^{k-1} b^{q-1} m_{k-1, q-1}(t-\tau)^{-(k-1) / \alpha}+ \\
& +\frac{k(k-1)}{2} q(q-1) B^{k-2} b^{q-2} m_{k-2, q-2} \times \\
& \left.\times(t-\tau)^{-(k-2) / \alpha}+\ldots\right] e^{-c_{0}^{\prime}(t-\tau)|\sigma|^{\alpha}} \leq \\
& \leq c_{2} B^{k} b^{q} m_{k q}(t-\tau)^{-k / \alpha} \times \\
& \times\left[1+\frac{T^{1 / \alpha}}{b B} k q \frac{m_{k-1, q-1}}{m_{k q}}+\frac{1}{2} \frac{T^{2 / \alpha}}{b^{2} B^{2}} \times\right. \\
& \left.\times k q \frac{m_{k-1, q-1}}{m_{k q}}(k-1)(q-1) \frac{m_{k-2, q-2}}{m_{k-1, q-1}}+\cdots\right] \times \\
& \times e^{-c_{0}^{\prime}(t-\tau)|\sigma|^{\alpha}} \leq \\
& \leq c_{2} B^{k} b^{q} m_{k q}(t-\tau)^{-\frac{k}{\alpha}}\left[1+\frac{\gamma T^{1 / \alpha}}{b B}(k+q)+\right. \\
& \left.+\frac{\gamma^{2} T^{2 / \alpha}}{1 \cdot 2 \cdot b^{2} B^{2}}(k+q)^{2}+\ldots\right] e^{-c_{0}^{\prime}(t-\tau)|\sigma|^{\alpha}} \leq \\
& \leq c_{2} B_{1}^{k} b_{1}^{q} m_{k q}(t-\tau)^{-\mathrm{k} / \alpha} e^{-c_{0}^{\prime}(t-\tau)|\sigma|^{\alpha}}= \\
& =c_{2} B_{1}^{k} b_{1}^{q}(t-\tau)^{-k / \alpha} k^{k / \alpha} q^{q} e^{-c_{0}^{\prime}(t-\tau)|\sigma|^{\alpha}} \text {, }
\end{aligned}
$$

where $B_{1}=B e^{\gamma T^{1 / \alpha} /(b B)}, b_{1}=b e^{\gamma T^{1 / \alpha} /(b B)}$.

Consequently,

$$
\begin{gathered}
\left|x^{q} D_{x}^{k} G(t-\tau, x ; \chi, \xi)\right| \leq \\
\leq c_{2}(2 \pi)^{-1} B_{1}^{k} b_{1}^{q}(t-\tau)^{-k / \alpha} k^{k / \alpha} q^{q} \times \\
\times \int_{\mathbb{R}} \exp \left\{-c_{0}^{\prime}(t-\tau)|\sigma|^{\alpha}\right\} d \sigma \leq
\end{gathered}
$$$$
\leq c_{3} B_{1}^{k} b_{1}^{q}(t-\tau)^{-(k+1) / \alpha} k^{k / \alpha} q^{q},\{k, q\} \subset \mathbb{Z}_{+}
$$

Then

$$
\begin{gathered}
\left|D_{x}^{k} G(t-\tau, x ; \chi, \xi)\right| \leq \\
\leq c_{3} B_{1}^{k}(t-\tau)^{-(k+1) / \alpha} k^{k / \alpha} \inf _{q} \frac{b_{1}^{q} q^{q}}{|x|^{q}} \leq \\
\leq c_{4} B_{1}^{k}(t-\tau)^{-(k+1) / \alpha} k^{k / \alpha} e^{-b_{0}|x|}, x \in \mathbb{R}, k \in \mathbb{Z}_{+},
\end{gathered}
$$

where $b_{0}=b_{1}^{-1}$. The following statement is correct.

Lemma 1. The function $G$, as a function of variable $x$, is an element of the space $S_{1}^{1 / \alpha}$. For functions $G$ and its derivatives (the variable $x$ ), the inequality

$$
\left|D_{x}^{k} G(t-\tau, x ; \chi, \xi)\right| \leq
$$

$\leq c_{4} B_{1}^{k}(t-\tau)^{-(k+1) / \alpha} k^{k / \alpha} e^{-b_{0}|x|}, k \in \mathbb{Z}_{+}, x \in \mathbb{R}$,

holds, constants $c_{4}, B_{1}, b_{0}>0$ are independent of $t-$

$\tau, \chi, \xi$.

The function 


$$
\begin{aligned}
G(t-\tau, x ; \chi, \xi)= & (2 \pi)^{-1} \int_{\mathbb{R}} Q(t \\
& -\tau, \chi ; \xi, \sigma) e^{-i x \sigma} d \sigma \quad(8)
\end{aligned}
$$

is a continuous function of the argument $t \in(\tau, T]$. Indeed, it follows from (7) that for $t \geq t_{0}>\tau$ true assessment $|Q(t-\tau, \chi ; \xi, \sigma)| \leq \exp \left\{-\left(t_{0}-\tau\right) c_{0}^{\prime}|\sigma|^{\alpha}\right\}, \quad \sigma \in \mathbb{R}$.

From this we already see that the integral (8) coincides uniformly in an arbitrary band $\left\{(t, \sigma): \tau<t_{0} \leq t \leq T\right.$, $\sigma \in \mathbb{R}\}$, so the function $G$ is continuous at every point in the interval $(\tau, T]$. Similarly, the differentiability of $G$ by the variable $t$ is proved.

Let $G_{0}=G(t-\tau, x ; \tau, 0), \varphi \in W_{M_{1}}^{\Omega_{1}}(\mathbb{R}) \subset S_{1}^{1}$. Taking advantage of the continuity property of the Fourier transform in $S$ spaces and the formula

$$
\begin{gathered}
F\left[\varphi * G_{0}\right]=F[\varphi] \cdot F\left[G_{0}\right]=F[\varphi] \cdot Q_{0}, \\
Q_{0}=Q(t-\tau, \tau ; 0, \sigma)
\end{gathered}
$$

we find that

$$
\begin{gathered}
\mu_{t \rightarrow \tau+0} F\left[\varphi * G_{0}\right]-\sum_{k=1}^{m} \mu_{k} \lim _{t \rightarrow t_{k}} F\left[\varphi * G_{0}\right]= \\
=F[\varphi]\left(\mu \lim _{t \rightarrow \tau+0} Q_{0}-\sum_{k=1}^{m} \mu_{k} \lim _{t \rightarrow t_{k}} Q_{0}\right) .
\end{gathered}
$$

Note that

$$
\mu \lim _{t \rightarrow \tau+0} Q_{0}-\sum_{k=1}^{m} \mu_{k} \lim _{t \rightarrow t_{k}} Q_{0}=1 .
$$

Then

$$
\mu \lim _{t \rightarrow \tau+0} F\left[\varphi * G_{0}\right]-\sum_{k=1}^{m} \mu_{k} \lim _{t \rightarrow t_{k}} F\left[\varphi * G_{0}\right]=F[\varphi] .
$$

Consequently,

$$
\mu \lim _{t \rightarrow \tau+0}\left(\varphi * G_{0}\right)-\sum_{k=1}^{m} \mu_{k} \lim _{t \rightarrow t_{k}}\left(\varphi * G_{0}\right)=\varphi .
$$

Since, on the other hand,

$$
\varphi * G_{0}=\int_{\mathbb{R}} G(t-\tau, x-\xi ; \tau, 0) \varphi(\xi) d \xi,
$$

then for an arbitrary function $\varphi \in W_{M_{1}}^{\Omega_{1}}(\mathbb{R})$ true value

$$
\begin{gathered}
\mu \lim _{t \rightarrow \tau+0} \int_{\mathbb{R}} G(t-\tau, x-\xi ; \tau, 0) \varphi(\xi) d \xi- \\
-\sum_{k=1}^{m} \mu_{k} \lim _{t \rightarrow t_{k}} \int_{\mathbb{R}} G(t-\tau, x-\xi ; \tau, 0) \varphi(\xi) d \xi= \\
=\varphi(x)(9)
\end{gathered}
$$

at each point $x \in \mathbb{R}$. Note that (9) implies a relation

$$
\begin{gathered}
\mu \lim _{t \rightarrow \tau+0} \int_{\mathbb{R}} G(t-\tau, x-\xi ; \tau, \xi) \varphi(\xi) d \xi- \\
-\sum_{k=1}^{m} \mu_{k} \lim _{t \rightarrow t_{k}} \int_{\mathbb{R}} G(t-\tau, x-\xi ; \tau, \xi) \varphi(\xi) d \xi \\
=\varphi(x)(10)
\end{gathered}
$$

at each point $x \in \mathbb{R}$ for an arbitrary function $\varphi \in$ $W_{M_{1}}^{\Omega_{1}}(\mathbb{R})$.

From the above results we also get that $\quad G(t-$ $\tau, x-\xi ; \tau, \xi$ ), as a function of $t, x$ (for fixed $\tau, \xi$ ), is the solution of equation (1). Therefore, $G(t-\tau, x-\xi ; \tau, \xi)$ can be taken as a function of $V(t, x ; \tau, \xi)$.

Let

$$
\begin{gathered}
I(t, \tau, x):= \\
=\int_{\tau}^{t} d \mu \int_{\mathbb{R}} G(t-\mu, x-\xi ; \mu, \xi) \varphi(\mu, \xi) d \xi,
\end{gathered}
$$

where $\varphi(t, x)$ is a function set to $[0, T] \times \mathbb{R}$, continuous on $t, \varphi(t, \cdot) \in W_{M_{1}}^{\Omega_{1}}(\mathbb{R})$ for every $t \in$ $[0, T]$. The following statement gives the formula for applying the operator $\partial / \partial t$ to the integral (11).

Lemma 2. The following formula holds:

$$
\begin{gathered}
\frac{\partial I(t, \tau, x)}{\partial t}= \\
=\int_{\tau}^{t} d \mu \int_{\mathbb{R}} \frac{\partial}{\partial t} G(t-\mu, x-\xi ; \mu, \xi) \varphi(\mu, \xi) d \xi+ \\
+\varphi(t, x) .(12)
\end{gathered}
$$

Proof. Consider the family of functions $\left\{I_{h}(t, \tau, x)\right.$, $0<h<t-\tau\}$, where

$$
\begin{gathered}
I_{h}(t, \tau, x)= \\
=\int_{\tau}^{t-h} d \mu \int_{\mathbb{R}} G(t-\mu, x-\xi ; \mu, \xi) \varphi(\mu, \xi) d \xi \equiv \\
\equiv \int_{\tau}^{t-h} g(t, \mu, x) d \mu, \\
g(t, \mu, x)=\int_{\mathbb{R}} G(t-\mu, x-\xi ; \mu, \xi) \varphi(\mu, \xi) d \xi .
\end{gathered}
$$

Applying the Lopital rule of differentiation of integrals depending on parameters, we find that

$$
\begin{gathered}
\frac{\partial I_{h}(t, \tau, x)}{\partial t}=\int_{\tau}^{t-h} \frac{\partial}{\partial t} g(t, \mu, x) d \mu+g(t, t-h, x)= \\
=\int_{\tau}^{t-h} d \mu \int_{\mathbb{R}} \frac{\partial}{\partial t} G(t-\mu, x-\xi ; \mu, \xi) \varphi(\mu, \xi) d \xi+ \\
\quad+\int_{\mathbb{R}} G(h, x-\xi ; t-h, \xi) \varphi(t-h, \xi) d \xi .
\end{gathered}
$$

We prove that $\left\{I_{h}, 0<h<t-\tau\right\}$ coincides at $h \rightarrow 0$ with the function $I(t, \tau, x)$, and $\quad\left\{\frac{\partial I_{h}}{\partial t}, 0<h<t-\right.$ $\tau\}$ coincides with $h \rightarrow 0$ uniformly with respect to $t$ the right side (12). Then, using the corresponding theorem from the mathematical analysis, we obtain that the function $I(t, \tau, x)$ is the differential of $t$, thus the equality (12) holds.

From Lemma 1, the estimate follows

$$
\begin{gathered}
|G(t-\mu, x-\xi ; \mu, \xi)| \leq \\
\leq c_{0}(t-\tau)^{-1 / \alpha} \exp \left\{-b_{0}|x-\xi|\right\} .
\end{gathered}
$$

Since $\sup _{\mu \in[0, T]}|\varphi(\mu, \xi)| \leq c, \forall \xi \in \mathbb{R}$, then

$$
\begin{aligned}
& \int_{\mathbb{R}}|G(t-\mu, x-\xi ; \mu, \xi)| \cdot|\varphi(\mu, \xi)| d \xi \leq \\
& \leq \tilde{c}(t-\mu)^{-1 / \alpha} \int_{\mathbb{R}} \exp \left\{-b_{0}|x-\xi|\right\} d \xi=
\end{aligned}
$$




$$
\begin{gathered}
=c^{\prime}(t-\mu)^{-1 / \alpha}, \\
c^{\prime}=\tilde{c} \int_{\mathbb{R}} \exp \left\{-b_{0}|y|\right\} d y=2 \tilde{c}_{0} b_{0}^{-1} .
\end{gathered}
$$

Hence we get that

$$
\begin{gathered}
\left|I_{h}(t, \tau, x)-I(t, \tau, x)\right| \leq c^{\prime} \int_{t-h}^{\tau}(t-\mu)^{-1 / \alpha} d \mu= \\
=\frac{h^{1-1 / \alpha}}{1-1 / \alpha}, \quad 1-1 / \alpha>0, \quad \alpha>2,
\end{gathered}
$$

that is $\lim _{h \rightarrow 0} I_{h}(t, \tau, x)=I(t, \tau, x)$. Let

$$
\begin{gathered}
\beta_{h}(t, x):= \\
=\int_{\tau}^{t-h} d \mu \int_{\mathbb{R}} \frac{\partial}{\partial t} G(t-\mu, x-\xi ; \mu, \xi) \varphi(\mu, \xi) d \xi, \\
\beta(t, x):= \\
=\int_{\tau}^{t} d \mu \int_{\mathbb{R}} \frac{\partial}{\partial t} G(t-\mu, x-\xi ; \mu, \xi) \varphi(\mu, \xi) d \xi .
\end{gathered}
$$

Since

$$
\begin{gathered}
\frac{\partial}{\partial t} G(t-\mu, x-\xi ; \mu, \xi)=(2 \pi)^{-1} \times \\
\times \int_{\tau}^{t-h} a(\mu, \xi ; \sigma) Q(t-\mu, \mu ; \xi, \sigma) e^{-i \sigma(x-\xi)} d \xi,
\end{gathered}
$$

then, taking into account the method of estimating $|G|$ (see proof of Lemma 1) and the properties of the symbolfunction $a$ (see condition 2)), it is found that

$$
\begin{array}{r}
\left|\frac{\partial}{\partial t} G(t-\mu, x-\xi ; \mu, \xi)\right| \leq \\
\leq c_{0}^{\prime}(t-\mu)^{-1 / \alpha} \exp \{-\bar{a}|x-\xi|\},
\end{array}
$$

where constants $c_{0}^{\prime}, \bar{a}>0$ are independent of $\mu, \xi$; in this case, for the integrand $|a(\mu, \xi ; \sigma) Q(t-\mu, \mu ; \xi, \sigma)|$ the inequality holds $\Lambda \leq \tilde{b} \exp \{M(\varepsilon \sigma)-M(a(t-\mu) \sigma)\}$, where $\varepsilon>0$ is an arbitrarily fixed parameter. The convexity property of $M$ implies inequalities

$$
\begin{gathered}
\exp \{M(\varepsilon \sigma)-M(a(t-\mu) \sigma)\} \leq \\
\leq \exp \{-M((a(t-\mu)-\varepsilon) \sigma)\}= \\
=\exp \left\{-M\left(\frac{a}{2}(t-\mu) \sigma\right)\right\} \leq \exp \left\{-d_{0}(t-\mu)|\sigma|^{\alpha}\right\},
\end{gathered}
$$

if we set $\varepsilon=a(t-\mu) / 2$. Further proof of (13) is carried out under the scheme prove estimates for the $|G|$.

Taking into account (13) and the inequality $\sup |\varphi(\mu, \xi)| \leq c$ find that

$$
\begin{gathered}
\int_{\mathbb{R}}\left|\frac{\partial}{\partial t} G(t-\mu, x-\xi ; \mu, \xi)\right| \cdot|\varphi(\mu, \xi)| d \xi \leq \\
\leq L(t-\mu)^{-1 / \alpha},
\end{gathered}
$$

where constant $L>0$ is independent of $t, \mu, x$. It follows that

$$
\begin{gathered}
\left|\beta_{h}(t, x)-\beta(t, x)\right| \leq L \int_{t-h}^{t}(t-\mu)^{-1 / \alpha} d \mu= \\
=\frac{L h^{1-1 / \alpha}}{1-1 / \alpha} \rightarrow 0
\end{gathered}
$$

at $h \rightarrow 0$ uniformly with respect to $t$.

From the results given in Lemma 1, it follows that for $G(h, x-\xi ; t-h, \xi)$ the estimate is correct:

$$
|G(h, x-\xi ; t-h, \xi)| \leq c h^{-1 / \alpha} \exp \left\{-b_{0}|x-\xi|\right\},
$$

which is uniform with respect to $t$. Hence, from relation (10) (which should be considered $\mu=1, \mu_{1}=\ldots=\mu_{m}=$ $0)$ and the continuity properties of functions $\varphi(t, x)$ by the variable $t$ implies that

$$
\begin{gathered}
\int_{\mathbb{R}} G(h, x-\xi ; t-h, \xi) \varphi(t-h, \xi) d \xi \rightarrow \varphi(t, x), \\
h \rightarrow 0,
\end{gathered}
$$

is uniform with respect to $t$. This proved that the family of functions $\left\{\partial I_{h} / \partial t, 0<h<t-\tau\right\}$ coincides with $h \rightarrow 0$ uniformly respect to $t$ to the right side (12). The proof of the lemma is complete.

Further operator $A$ in equation (1) we understand as an operator acting from the space $X$ in $K(\mathbb{R})$, where the symbol $X$ denote the space consisting of functions $\psi \in$ $W_{M_{1}}^{\Omega_{1}}(\mathbb{R})$ with norm $\|\psi\|==\sup _{x \in \mathbb{R}}|\psi(x)|$.

Lemma 3. 1. Let $\varphi(t, x),(t, x) \in[0, T] \times \mathbb{R}$, be a function, continuous on the variable $t, \quad \varphi(t, \cdot) \in$ $W_{M_{1}}^{\Omega_{1}}(\mathbb{R})$. The following formula holds:

$$
\begin{aligned}
A I(t, \tau, x)=\int_{\tau}^{t} d \mu \int_{\mathbb{R}} A G(t-\mu, x \\
-\xi ; \mu, \xi) \varphi(\mu, \xi) d \xi
\end{aligned}
$$

2. The estimate for $A G$ is correct

$$
|A G(t-\mu, x-\xi ; \mu, \xi)| \leq
$$$$
\leq c(t-\mu)^{-1 / \alpha} \exp \{-a|x-\xi|\}, \quad t>\mu \geq 0,
$$

constant $c, a>0$ is independent of $t, \mu$.

The proof of Lemma 3 is carried out according to the scheme of proof of Lemma 2.

On the basis of Lemmas 2, 3, we conclude that with the above restrictions on the function $\varphi$ the following formula holds:

$$
L I(t, \tau, x)=\int_{\tau}^{t} d \mu \times
$$$$
\times \int_{\mathbb{R}} L G(t-\mu, x-\xi ; \mu, \xi) \varphi(\mu, \xi) d \xi+\varphi(t, x),
$$

the $L G$ function satisfies the inequality

$$
\begin{gathered}
|L G(t-\tau, x-\xi ; \tau, \xi)| \leq \\
\leq c(t-\tau)^{-1 / \alpha} \exp \{-a|x-\xi|\},(15)
\end{gathered}
$$

where $c, a>0$ is independent of $t, \tau, t>\tau$.

Let us now proceed to constructing a fundamental solution of a multi-point problem for equation (1); this solution looking as the sum of:

$Z(t, x ; \tau, \xi)=G(t-\tau, x-\xi ; \tau, \xi)+\Gamma(t, x ; \tau, \xi),(16)$

$(t, x) \in \Pi^{\prime}{ }_{T}$, where

$$
\begin{gathered}
\Gamma(t, x ; \tau, \xi)= \\
=\int_{\tau}^{t} d \mu \int_{\mathbb{R}} G(t-\mu, x-\eta ; \mu, \eta) \Phi(\mu, \eta ; \tau, \xi) d \eta
\end{gathered}
$$

$G$ is a function defined earlier. We choose the function $\Phi(t, x ; \tau, \xi)$ so that $Z$, as a function of $t, x$, satisfies equation (1). Applying the operator $L$ to $Z$ and taking into account formulas (12), (14), we find that this will be the case if and only if

$$
\begin{gathered}
\Phi(t, x ; \tau, \xi)=K(t-\tau, x ; \tau, \xi)+ \\
+\int_{\tau}^{t} d \mu \int_{\mathbb{R}} K(t-\mu, x ; \mu, \eta) \Phi(\mu, \eta ; \tau, \xi) d \eta,
\end{gathered}
$$


where $K(t-\tau, x ; \tau, \xi)=-L G(t-\tau, x-\xi ; \tau, \xi)$. The series

$$
\begin{gathered}
\Phi(t, x ; \tau, \xi)=\sum_{m=1}^{\infty} K_{m}(t-\tau, x ; \tau, \xi) \\
K_{1}=K, K_{m}(t-\tau, x ; \tau, \xi)= \\
=\int_{\tau}^{t} d \beta \int_{\mathbb{R}} K(t-\beta, x ; \beta, \eta) K_{m-1}(\beta-\tau, \eta ; \tau, \xi) d \eta,
\end{gathered}
$$

is a formal solution of the integral equation (18). We examine series (19) for absolute and uniform convergence at $0<\delta_{0} \leq t-\tau \leq T$. To justify the convergence of this series, let us evaluate the kernels of $K_{m}$. Note that $\left|K_{1}\right|=$ $|L G|$ holds (15). To evaluate the kernel $K_{2}$, we use the following auxiliary statement.

Let

$$
I(x, \xi):=\int_{\mathbb{R}} \exp \{-a(|x-y|+|y-\xi|)\} d y, a>0 .
$$

For integral $I$ true inequality

$$
I(x, \xi) \leq c(\varepsilon) \exp \{-a(1-\varepsilon)|x-\xi|\},(20)
$$

where $0<\varepsilon<1$ is a fixed parameter, and $c(\varepsilon)=$ $2 a^{-1} \varepsilon^{-1}$. Indeed, consider the function $\varphi(y)=|x-y|+$ $|y-\xi|$. We are convinced that it satisfies the inequality $\varphi(y) \geq|x-\xi|$. Fixed $\quad 0<\varepsilon<1$. Then

$$
\begin{gathered}
I(x, \xi)= \\
=\exp \{-a(1-\varepsilon)|x-\xi|\} \int_{\mathbb{R}} \exp \{-a \varepsilon \varphi(y)\} d y= \\
=\exp \{-a(1-\varepsilon)|x-\xi|\} \int_{\mathbb{R}} \exp \{-a \varepsilon|y-\xi|\} d y= \\
=c(\varepsilon) \exp \{-a(1-\varepsilon)|x-\xi|\},
\end{gathered}
$$

where $c(\varepsilon)=2 a^{-1} \varepsilon^{-1}$. Taking into account (15) and (20), we estimate the kernel $K_{2}$. So,

$$
\begin{aligned}
& \left|K_{2}(t-\tau, x ; \tau, \xi)\right| \leq \int_{\tau}^{t} d \beta \times \\
& \times \int_{\mathbb{R}}|K(t-\beta, x ; \beta, \eta)| \cdot\left|K_{1}(\beta-\tau, \eta ; \tau, \xi)\right| d \eta \leq \\
& \leq c^{2} \int_{\tau}^{t}\left(\int_{\mathbb{R}}(t-\beta)^{-\lambda}(\beta-\tau)^{-\lambda} \times\right. \\
& \left.\times e^{-a(|x-\eta|+|\eta-\xi|)}\right) d \beta \leq \\
& \leq c^{2} \int_{\tau}^{t}(t-\beta)^{-\lambda}(\beta-\tau)^{-\lambda} d \beta \times \\
& \times \int_{\mathbb{R}} e^{-a(|x-\eta|+|\eta-\xi|)} d \eta \leq \\
& \leq c^{2} c(\varepsilon)(t-\tau)^{1-2 \lambda} B(1-\lambda, 1-\lambda) \times \\
& \times \exp \{-a(1-\varepsilon)|x-\xi|\}, \quad \lambda=1 / \alpha ; \quad \text { (21) } \\
& \begin{array}{l}
\int_{a}^{b}(t-a)^{x-1}(b-t)^{y-1} d t= \\
a)^{x+y-1} B(x, y), \quad \operatorname{Re} x>0, \text { Re } y>0,
\end{array}
\end{aligned}
$$

$B(\cdot, \cdot)$ is a beta-function. Taking into account $(21)$, we estimate the kernel $K_{3}$ :

$$
\begin{gathered}
\left|K_{3}(t-\tau, x ; \tau, \xi)\right| \leq \int_{\tau}^{t} d \beta \times \\
\times \int_{\mathbb{R}}|K(t-\beta, x ; \beta, \eta)| \cdot\left|K_{2}(\beta-\tau, \eta ; \tau, \xi)\right| d \eta \leq \\
\leq c^{3} c(\varepsilon) B(1-\lambda, 1-\lambda) \times \\
\times \int_{\tau}^{t}(t-\beta)^{-\lambda}(\beta-\tau)^{1-2 \lambda} \times \\
\quad \times\left(\int_{\mathbb{R}} e^{-a(|x-\eta|+(1-\varepsilon)|\eta-\xi|)} d \eta\right) d \beta \\
\text { Introduce: } \varphi(\eta)=|x-\eta|+|\eta-\xi| . \text { Then } \\
\left|K_{3}(t-\tau, x ; \tau, \xi)\right| \leq c^{3} c(\varepsilon) B(1-\lambda, 1-\lambda) \times \\
\quad \times \int_{\tau}^{t}(t-\beta)^{-\lambda}(\beta-\tau)^{1-2 \lambda} d \beta \times \\
\times \int_{\mathbb{R}} \mid \exp \{-a(1-\varepsilon) \varphi(\eta)-a \varepsilon|x-\eta|\} d \eta
\end{gathered}
$$

Since $\varphi(\eta) \geq|x-\xi|$, then

$$
\int_{\mathbb{R}} e^{-a(1-\varepsilon) \varphi(\eta)-a \varepsilon|x-\eta|} d \eta \leq c(\varepsilon) e^{-a(1-\varepsilon)|x-\xi|},
$$

where $c(\varepsilon)=\int_{\mathbb{R}} \exp \{-a \varepsilon|x-\eta|\} d \eta=2(a \varepsilon)^{-1} . \quad$ In addition,

$$
\begin{aligned}
\int_{\tau}^{t}(t-\beta)^{-\lambda}(\beta-\tau)^{1-2 \lambda} d \beta= & \\
= & (t-\tau)^{2-3 \lambda} B(1-\lambda, 2-2 \lambda)
\end{aligned}
$$

Therefore, $\left|K_{3}\right|$ is estimated as follows:

$$
\begin{gathered}
\left|K_{3}(t-\tau, x ; \tau, \xi)\right| \leq \\
\leq c^{3} c^{2}(\varepsilon) B(1-\lambda, 1-\lambda) B(1-\lambda, 2-2 \lambda) \times \\
\times(t-\tau)^{2-3 \lambda} \exp \{-a(1-\varepsilon)|x-\xi|\} .
\end{gathered}
$$

Using the method of mathematical induction prove that

$$
\begin{gathered}
K_{m}(t-\tau, x ; \tau, \xi) \mid \leq c^{m} c^{m-1}(\varepsilon) \times \\
\times B(1-\lambda, 1-\lambda) B(1-\lambda, 2-2 \lambda) \times \\
\times B(1-\lambda, 3-3 \lambda) \times \ldots \times \\
\times B(1-\lambda,(m-1)-(m-1) \lambda)(t-\tau)^{m-1-m \lambda} \times \\
\times \exp \{-a(1-\varepsilon)|x-\xi|\}, m \geq 2 .
\end{gathered}
$$

Taking into account formulas

$$
B(z, \omega)=\Gamma(z) \Gamma(\omega) / \Gamma(z+\omega)
$$

( $\Gamma$ is a gamma-function), $\Gamma(1+x)=x \Gamma(x)$ we find that

$$
\begin{gathered}
B(1-\lambda, 1-\lambda) B(1-\lambda, 2-2 \lambda) \mathrm{B}(1-\lambda, 3-3 \lambda) \ldots \\
\times B(1-\lambda,(m-1)-(m-1) \lambda)= \\
=\frac{\Gamma(1-\lambda) \Gamma(1-\lambda) \Gamma(1-\lambda) \Gamma(2-2 \lambda)}{\Gamma(2(1-\lambda)) \Gamma(3(1-\lambda)} \times \\
\times \frac{\Gamma(1-\lambda) \Gamma(3-3 \lambda) \ldots \Gamma((m-1)(1-\lambda))}{\Gamma(4(1-\lambda)) \ldots \Gamma(m(1-\lambda))}= \\
=\Gamma(1-\lambda) \frac{\Gamma^{m}(1-\lambda)}{\Gamma(m(1-\lambda))}, m \geq 2 .
\end{gathered}
$$
hold:

Thus, for the series $\sum_{m=0}^{\infty} K_{m}$, the following estimates 


$$
\begin{aligned}
& \left|\sum_{m=1}^{\infty} K_{m}(t-\tau, x ; \tau, \xi)\right| \leq \sum_{m=1}^{\infty}\left|K_{m}(t-\tau, x ; \tau, \xi)\right| \leq \\
& \leq c(t-\tau)^{-\lambda} e^{-a|x-\xi|}+c^{-1}(\varepsilon) \Gamma(1-\lambda)(t-\tau)^{-\lambda} \times \\
& \quad \times \sum_{m=2}^{\infty} c^{m} c^{m}(\varepsilon)(t-\tau)^{m(1-\lambda)} \times \\
& \quad \times \frac{\Gamma^{m}(1-\lambda)}{\Gamma(m(1-\lambda))} e^{-a(1-\varepsilon)|x-\xi|} \leq \\
& \leq c(t-\tau)^{-\lambda} e^{-a|x-\xi|}+c^{-1}(\varepsilon) \Gamma(1-\lambda)(t-\tau)^{-\lambda} \times \\
& \times \sum_{m=2}^{\infty} c^{m} c^{m}(\varepsilon) T^{m(1-\lambda)} \frac{\Gamma^{m}(1-\lambda)}{\Gamma(m(1-\lambda))} e^{-a(1-\varepsilon)|x-\xi|} .
\end{aligned}
$$

Because of the Stirling formula $\Gamma(x)=$ $\sqrt{2 \pi} e^{x} x^{x-\frac{1}{2}} e^{\frac{\theta}{12 x}}, x>0,0<\theta<1$, we have that

$$
\begin{gathered}
\frac{\Gamma^{m}\left(\omega_{0}\right)}{\Gamma\left(m \omega_{0}\right)} \leq \beta_{0} \frac{\theta_{0}^{m}}{m^{m \omega_{0}}}, \quad \beta_{0}=\frac{1}{\sqrt{2 \pi}}, \\
\theta_{0}=2 \sqrt{2 \pi} e, \quad \omega_{0}=1-\lambda .
\end{gathered}
$$

The last estimate implies the convergence of a series

$$
\sum_{m=2}^{\infty} \beta^{m} \frac{\Gamma^{m}(1-\lambda)}{\Gamma(m(1-\lambda))}, \quad \beta=c \cdot c(\varepsilon) T^{1-\lambda} .
$$

Since, the series $\sum_{m=1}^{\infty} K_{m}$ for $0<\delta_{0} \leq t-\tau \leq T$ coincides absolutely and evenly, and its sum is a function of $\Phi(t, x ; \tau, \xi)$ for $t>\tau$ is a continuous function of the arguments $x$, $\xi$. Set $\varepsilon=1 / 2$; then inequality holds for $\Phi$

$$
\begin{gathered}
|\Phi(t, x ; \tau, \xi)| \leq \\
\leq d_{0}(t-\tau)^{-\lambda} \exp \left\{-\frac{a}{2}|x-\xi|\right\} .
\end{gathered}
$$

This estimate ensures the convergence of integrals (17), (18). It follows that the integral in (18) is equal

$$
\begin{gathered}
\sum_{m=1}^{\infty} \int_{\tau}^{t} d \mu \int_{\mathbb{R}} K(t-\mu, x ; \mu, \eta) K_{m}(\mu-\tau, \eta ; \tau, \xi) d \eta= \\
=\sum_{m=1}^{\infty} K_{m+1}(t-\tau, x ; \tau, \xi) .
\end{gathered}
$$

So, $\Phi$ is the solution of equation (18).

Recall that the correct estimate for $|G|$ is

$$
|G(t-\tau, x-\xi ; \tau, \xi)| \leq
$$

$$
\leq c(t-\tau)^{-\lambda} \exp \{-a|x-\xi|\}, \quad \lambda=1 / \alpha, \quad \text { (23) }
$$

where constants $c, a>0$ are independent of $t, \tau$ (see Lemma 1). On the basis of inequalities (23), (22), (20), we estimate $\Gamma$; while in (20) put $\varepsilon=1 / 2$. So,

$$
|\Gamma(t, x ; \tau, \xi)| \leq \int_{\tau}^{t} d \mu \times
$$

$$
\begin{gathered}
\times \int_{\mathbb{R}_{t}}|G(t-\mu, x-\eta ; \mu, \eta)| \cdot|\Phi(\mu, \eta ; \tau, \xi)| d \eta \leq \\
\leq c \int_{\tau}(t-\mu)^{-\lambda}(\mu-\tau)^{-\lambda} \times \\
\times\left(\int_{\mathbb{R}} e^{-a|x-\eta|-\frac{a}{2}|\eta-\xi|} d \eta\right) d \mu \leq \\
\leq \tilde{c}(t-\tau)^{1-2 \lambda} \exp \left\{-\frac{a}{4}|x-\xi|\right\} .
\end{gathered}
$$

From estimate (24) implies that for any continuous bounded on $\mathbb{R}$ function $\varphi$ is

$$
\begin{gathered}
\int_{\mathbb{R}}|\Gamma(t, x ; 0, \xi)| \cdot|\varphi(\xi)| d \xi \leq \tilde{c} t^{1-2 \lambda} \times \\
\times \int_{\mathbb{R}} \exp \left\{-\frac{a}{4}|x-\xi|\right\} d \xi=d_{1} t^{1-2 \lambda}, 1-2 \lambda>0 .
\end{gathered}
$$

From this we already get that at every point $x \in \mathbb{R}$ the boundary relation holds

$$
\lim _{t \rightarrow+0} \int_{\mathbb{R}} \Gamma(t, x ; 0, \xi) \varphi(\xi) d \xi=0 .
$$

On the basis of the obtained results, we claim that the function

$$
\begin{gathered}
Z(t, x ; \tau, \xi)=V(t, x ; \tau, \xi)+\Gamma(t, x ; \tau, \xi), \\
V(t, x ; \tau, \xi)=G(t-\tau, x-\xi ; \tau, \xi),
\end{gathered}
$$

is a fundamental solution of the nonlocal $m$-point in time problem for equation (1), and the function

$$
\begin{gathered}
u(t, x)= \\
=\int_{\mathbb{R}} \mathrm{V}(t, x ; 0, \xi) \varphi(\xi) d \xi+\int_{\mathbb{R}} \Gamma(t, x ; 0, \xi) \varphi(\xi) d \xi= \\
=u_{1}(t, x)+u_{2}(t, x), \quad \text { (25) }
\end{gathered}
$$

$(t, x) \in(0, T] \times \mathbb{R}, \varphi \in W_{M_{1}}^{\Omega_{1}}(\mathbb{R})$, is the solution of this problem at $\tau=0$. We summarize the results obtained in the form of the following statement.

Theorem. $m$-point problem for equation (1) with parameter $\tau=0$ is solvable in class $X$, with the solution given by formula $(25) ; u(t, x)$ is a continuous function of $x$ bound for $\mathbb{R}$ for every $t \in(0, T]$.

Conclusions. A solution of nonlocal in time multi-point problem for evolution equation with a pseudodifferential operator constructed at variable symbols in a class of bounded continuous on $\mathbb{R}$ functions is established. The definitions and properties of the fundamental solution of this problem are given.

\section{REFERENCES}

1. A.M. Nakhushev. Equations of mathematical biology [in Russian], Higher School, Moscow (1995).

2. I.A. Belavin, S.P. Kapitsa, S.P. Kurdyumov. Mathematical model of global demographic processes taking into account spatial distribution [in Russian], Journal will calculate. Mat. and mat. physics, (1988).

3. A.A. Desin. General questions of boundary value theory [in Russian], Science, Moscow (1980).

4. V.K. Romanko. Boundary value problems for one class of differential operators [in Russian], Differential equations, (1974), Vol. 10, N11.

5. V.K. Romanko. Nonlocal boundary value problems for some systems of equations [in Russian], Mat. Notes, (1985), Vol. 37 ,
N7.

6. A.A. Makarov. Existence of a correct two-point boundary value problem in a layer for systems of pseudodifferential equations[in Russian], Differential equations, (1994), T. 30, N1.

7. V.I. Chesalin. A problem with nonlocal boundary conditions for abstract hyperbolic equations. [in Russian], Differential equations, (1979), Vol.15, N11.

8. V.S. Ilkiv, B.I. Ptashnik. Some non-local two-point problem for systems of partial differential equations [in Russian], Sib. Mate, (2005), Vol.46, N1.

9. N.L. Lazetic. On classical solutions of mixed boundary problems for one-dimensional parabolic equation of second 
order. Pablications de Institut Mathematique, (2000). Vol.67.

10. Chabrowski J. On nonlocal problems with a functional for parabolic equation. Function Equation, (1984), Vol.27.

11. Bouziani A., Benouar N.E. Problems of mixed avec conditions integrales pour une class d'equations paraboliques. C.R. Acad. Sci. Paris. Sir. J. (1995).

12. I.M. Gelfand, G.E. Shilov. Some questions of the theory of differential equations [in Russian], Fizmatgiz, Moscow (1958).

13. B.L. Gurevich. Some spaces of basic and generalized functions and the Cauchy problem for finite difference schemes[in Russian], Doc. USSR Academy of Sciences,
(1954), T.99, N6.

14. I.M. Gelfand, G.E. Shilov. Spaces of basic and generalized functions [in Russian], Fizmatgiz, Moscow (1958).

15. V.V. Gorodetsky, O.M. Leniuk. The Cauchy problem for evolution equations with infinitely differentiating operators [in Ukrainian], Bulletin of the Kiev Univ. Series: Phys. Science, (2000) Issue 4.

16. V.V. Gorodetsky, O.V. Martyniuk. Evolutionary pseudodifferential equations in numerically normalized spaces [in Ukrainian], Techno-print, Chernivtsi (2016). 